\section{BMJ Paediatrics Open}

\title{
Integrating eye health training into the primary child healthcare programme in Tanzania: a pre-training and post- training study
}

\author{
Aeesha N J Malik (D , ${ }^{1}$ Mlika Mafwiri, ${ }^{2}$ Clare Gilbert, ${ }^{1}$ Min J Kim, ${ }^{3}$ \\ Joanna Schellenberg ${ }^{1}$
}

\begin{abstract}
To cite: Malik ANJ, Mafwiri M, Gilbert C, et al. Integrating eye health training into the primary child healthcare programme in Tanzania: a pre-training and post-training study. BMJ Paediatrics Open 2020;4:e000629. doi:10.1136/ bmjpo-2019-000629
\end{abstract}

- Additional material is published online only. To view please visit the journal online (http://dx.doi.org/10.1136/ bmjpo-2019-000629).

Received 18 December 2019 Revised 27 May 2020 Accepted 30 May 2020

\section{Check for updates}

(c) Author(s) (or their employer(s)) 2020. Re-use permitted under CC BY-NC. No commercial re-use. See rights and permissions. Published by BMJ.

${ }^{1}$ International Centre of Eye Health, London School of Hygiene and Tropical Medicine, London, UK

${ }^{2}$ Department of Ophthalmology, Muhimbili University of Health and Allied Sciences, Dar es Salaam, United Republic of Tanzania

${ }^{3}$ Department of Infectious Disease Epidemiology, London School of Hygiene and Tropical Medicine, London, UK

Correspondence to Dr Aeesha N J Malik; aeesha. malik@|shtm.ac.uk

\section{ABSTRACT}

Objectives To evaluate a primary child eye care training module for use in the WHO/UNICEF Integrated Management of Newborn and Childhood IIIness (IMNCI) programme by primary healthcare workers.

Design Pre-training and post-training study.

Setting Primary healthcare facilities in a semirural district, central Tanzania.

Participants 54 primary healthcare workers selected by the Ministry of Health were trained during routine IMNCI training. All these healthcare workers were assessed preintervention and post-intervention. A subgroup of 40 were also assessed 6 months after the completion of training. Intervention Training in an IMNCl eye module, developed for child primary healthcare workers with the Tanzania Ministry of Health and eye care experts.

Main outcome measures Knowledge, skills and attitudes were assessed using multiple choice questions, case studies and a self-assessment of skills using a Likert scale before and immediately after training, and 6 months later. A total score was derived. At 6 months, attitudes were assessed in semistructured interviews.

Results $69 \%$ PHWs trained were nurses. The baseline (before training) score was 29.9 (95\% Cl 27.5 to 32.4$)$ and increased by 11.2 points $(95 \% \mathrm{Cl} 8.3$ to 14$)$ immediately after training, and by 12.4 points $(95 \% \mathrm{Cl} 9.2$ to 15.6$)$ at 6 months post the training. Therefore, the post-training scores increased and there was no evident difference in scores from immediately after training to 6 months later. Self-assessed confidence in skills decreased from $9 / 18$ ( $95 \% \mathrm{Cl} 9$ to 10 ) to $6 / 18$ (95\% Cl 6 to 7$)$. At 6 months, the module was reported as easy to understand and use, with challenges including difficulties in examining children's eyes and poor referral systems.

Conclusions The module increased knowledge of child eye health in primary healthcare workers, which was maintained, and was acceptable. The module has since been included into the national IMNCI health policy in Tanzania.

\section{INTRODUCTION}

Approximately $75 \%$ of the 1.2 million blind children in the world live in low-income and middle-income countries (LMICs), approximately half of whom are blind from avoidable
What is known about the subject?

Many children in low-income countries present too late for effective management, leading to avoidable blindness.

- There is a critical shortage of primary level eye care services for children in low-income countries.

- There are evidence-based primary level interventions which can prevent avoidable blindness in children but these are not being implemented by primary healthcare workers (PHWs) as they are not included in child health training programmes.

\section{What this study adds?}

Eye care training integrated into a child health training programme for primary healthcare workers improved their knowledge and case management of children's eye conditions.

- Including eye care into the child health training programme is acceptable to PHWs and they were able to implement it within their routine care.

- Tanzanian national health policy now includes eye care in the Integrated Management of Newborn and Childhood IIIness programme demonstrating it is a feasible model for scale-up in other countries.

causes that is, conditions which are either preventable or treatable. ${ }^{1}$ Preventable causes include corneal scarring from vitamin A deficiency, measles infection, conjunctivitis of the newborn and use of harmful eye remedies. As vitamin A deficiency and measles are declining due to public health interventions, treatable causes such as cataract, glaucoma and retinoblastoma are assuming greater importance. These conditions need to be detected and treated early, as delayed management leads to poorer outcomes due to delayed visual development. ${ }^{2-4}$ The prevalence of blindness in children aged $0-15$ years in low-income countries is approximately $0.1 \%$, with $80 \%$ 
of these children being either born blind or becoming blind before the age of 5 years. ${ }^{5}$ Eye care services for preschool age children are, therefore, a critical target for child health programmes in order to prevent life-long visual impairment, blindness and mortality.

The WHO has identified strengthening primary healthcare as a critical component of Universal Health Coverage throughout LMICs. ${ }^{6}$ In 2002, WHO identified 10 evidence-based 'key activities for healthy eyes in children' for primary care. These interventions include vitamin A supplementation and measles immunisation as well as ocular prophylaxis of the newborn and early screening for cataract. ${ }^{4} \mathrm{WHO}$ recommended these activities be included in primary level child-health services and national prevention of blindness plans. ${ }^{7}$ However, the eye-specific components have not been included in child health programmes. ${ }^{8}$

In 1995, WHO launched the Integrated Management of Childhood Illness (IMCI) which has become the blueprint for primary care of children in over 100 countries. $^{9}$ Care of the newborn has since been added (IMNCI). The goal of IMNCI is to reduce mortality, illness and disability, and promote growth and development. It includes prevention as well as treatment. Health worker training is conducted using modules which cover the management of conditions such as fever, diarrhoea and ear infections. However, IMNCI does not include an eye care module.

In Tanzania, $63 \%$ of live births occur in healthcare facilities, and the prevalence of blindness in children aged $0-15$ years is estimated to be $8 / 10000$ children, and likely higher in poor, rural communities. ${ }^{10}$ Tanzania has an active IMNCI programme with continuous training of primary healthcare workers (PHWs).

A pilot study conducted in Dar es Salaam showed that PHWs had poor knowledge of eye conditions in children which improved after a brief training and was retained at 1 year. ${ }^{11}$ Sixty-one per cent of trained staff knew how to manage purulent conjunctivitis compared with $30 \%$ of untrained staff (30 different staff in 15 clinics); $82 \%$ of those trained and $33 \%$ of those not trained were able to correctly diagnose a cataract. Leading on from this pilot work, the aim of the current study was to address the gap in primary eye care for children by developing and evaluating an eye module for PHWs which could be included in the IMNCI programme in Tanzania. This study reports how the eye module was developed and the findings of a pretraining and post-training assessment to ensure fidelity before advocating for wider adoption of the eye care module for PHWs.

\section{METHODS}

\section{Development of the eye module}

The eye module comprised a detailed manual, and additions to the Facilitator's Guide, User Log and 'Chart Book'. The latter comprises a series of algorithms which classifies the severity of conditions as severe, not severe or not present based on clinical signs and symptoms,

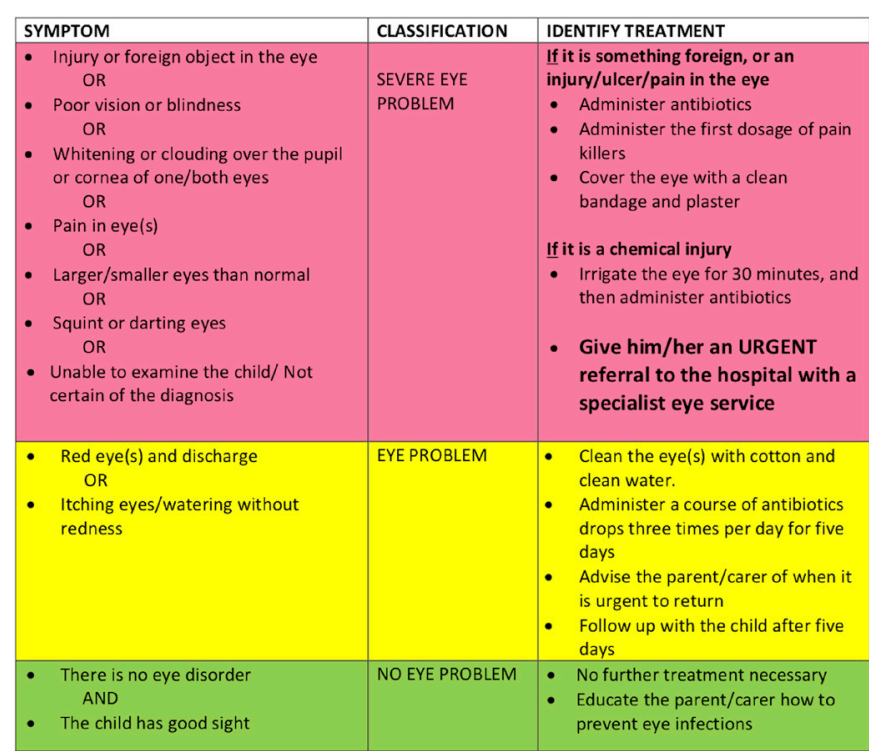

Figure 1 The classification of eye diseases summarised in the IMNCl 'chart' or algorithm. IMNCI, Integrated Management of Newborn and Childhood Illness.

and the management required (figure 1). A Steering Committee was convened for the study comprising stakeholders from the Ministry of Health, Community Development, Gender, Elderly and Children (referred to here as Ministry of Health), WHO, UNICEF, NonGovernmental Organisations and technical experts. A working group comprising IMNCI facilitators and technical experts developed the eye module in a series of meetings in Dar es Salaam. The manual was developed in Swahili and translated into English. The final version was reviewed and approved by the Ministry of Health in May 2018. The module included training on the Arclight, an innovative evidence-based low-cost ophthalmoscope to assess the red reflex for conditions such as cataract, which was given to every PHW. ${ }^{12}$

\section{PATIENT AND PUBLIC INVOLVEMENT}

This research was done without patient involvement. Patients were not invited to comment on the study design and were not consulted to develop patient relevant outcomes or interpret the results. Patients were not invited to contribute to the writing or editing of this document for readability or accuracy.

\section{STUDY POPULATION}

The study population comprised PHWs in dispensaries and health centres in Bahi district, Dodoma region. The PHWs comprised of registered and enrolled nurses who have at least 3 years nurse training and are the main providers of antenatal care, deliveries and care for children below 5 years. Clinical officers are trained for at least 2 years after secondary school education in general medical problems. Assistant medical officers are clinical officers who have completed at least 2 years 
further training mainly in clinical medicine for common diseases. Medical attendants have primary or secondary education and assist in supportive roles in healthcare facilities alongside the nurses and clinical officers.

The location was selected by the Ministry of Health as part of their routine IMNCI training schedule. Selection of participants for training was according to IMNCI guidelines to ensure at least $50 \%$ of PHWs delivering IMNCI were trained.

\section{Routine IMNCI training}

Routine IMNCI training was conducted using the WHO distance learning approach. ${ }^{13}$ Phase I has 1 day of faceto-face teaching, where the first six modules are introduced. The training is conducted by Ministry of Health IMNCI facilitators who are usually paediatricians from tertiary hospitals who have been trained as facilitators. The teaching includes formal presentations and teaching on each module to highlight important points and any recent changes in practice. This is followed by time for questions. There is a short skills training session at a nearby local health centre. PHWs are then allocated into small 'study' groups led by one of the IMNCI's facilitators. Over the next 6 weeks trainees study the modules alone and with their group, and put into practice what they have learnt. During this phase they have access to the IMNCI facilitator for advice. In phase II there is a further face-to-face 1 day meeting when the remaining modules are introduced, and there is time for questions arising from phase I and practise in their own setting. After another 6 weeks there is the third and final phase with a 1 day meeting to answer questions and summarise the main learning points. A final test is undertaken before certification.

\section{Assessment tools}

A range of tools were developed to assess the knowledge, skills and attitudes of PHWs in relation to eye conditions in children and how they should be prevented or managed at the primary level. All assessment tools were developed in English, translated into Swahili and backtranslated into English (online supplementary appendix 1). The tools were pretested on a group of 10 PHWs in Dar es Salaam and the findings were used to refine the assessment tools.

The tools included multiple choice questions (MCQs, 'True/False' and 'Best of 4') to test basic knowledge of eye conditions, identification of eye conditions from photographs ('Image' questions) and questions on common childhood eye conditions or scenarios which required written answers ('Case Studies'). This assessed knowledge of conditions, classification, management and referral criteria. A Likert scale was used for selfassessment of confidence in skills PHWs had in the examination, diagnosis and treatment of eye conditions in children ('Skills').

A semistructured topic guide with open-ended questions was developed for face-to-face interviews to seek

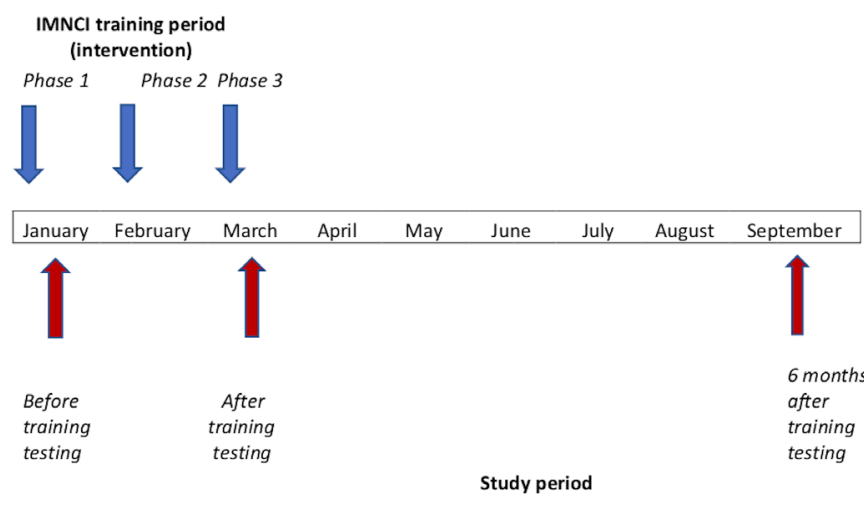

Figure 2 Study and intervention timeline 2018. IMNCI, Integrated Management of Newborn and Childhood Illness.

PHWs' views and attitudes towards the eye module in terms ease of use, level of content, applicability of use and challenges in daily practice. Interviews were conducted in Swahili by Tanzanian healthcare professionals at 6 months after the completion of training. Responses to questions were written down verbatim and translated into English.

The assessments were undertaken in the training facility immediately before phase I and after the final phase of the training period. Six months after completion of the training the PHWs were visited in their facilities where they completed the assessment tools again and the semistructured interviews took place (figure 2).

\section{Data management and analysis}

Data were entered into Microsoft Excel (v 2016) with precoded macros. Results from the questionnaires and the entries were checked by three members of the study team.

A total score was derived by the addition of the individual scores for the MCQs ('True/False' and 'Best of 4'), the 'Image' questions and 'Case Studies' which was then adjusted as a score out of 100 for ease of comparison. The MCQs were marked by awarding +1 for a correct score, -1 point for an incorrect answer and 0 for no answer. Likert scales for the 'Skills' assessment were analysed separately.

Qualitative responses were grouped under common themes by two members of the study team and reported verbatim with a narrative description of experiences and perceptions. The content was analysed and coded according to the responses to the main elements of the interviews (online supplementary appendix 2).

Fisher's exact test was used for categorical data and the rank sum test for continuous data to compare the characteristics and baseline scores of PHWs who were and who were not traced at 6 months. A multilevel mixed-effects linear regression analysis was used for analysis of the total score. The model used time (before, after and at 6 months postintervention) as an exposure with total score as an outcome. Random effects at person level were used to account for repeated measures at three time points of an individual. STATA (Release 16, StataCorp LLC, College Station, Texas, USA) was used for statistical analysis. 


\section{RESULTS}

Fifty-four PHWs were trained from January to March 2018. The majority were nurses $(37,69 \%), 19 \%$ (10) were clinical officers, $9 \%$ (5) were assistant medical officers and $4 \%$ (2) were medical attendants (table 1). A very high proportion $(48,89 \%)$ saw children with eye problems at least once a month, but only $5 \%$ (3) had been trained in eye care. The most common eye condition they encountered was conjunctivitis, followed by injuries or foreign bodies and corneal ulcers. Only 4\% (2) mentioned other conditions. However, 37\% (20) said they referred children with eye conditions for further medical advice at least once a month.

The mean total knowledge score improved from $40 \%$ (95\% CI $37 \%$ to $43 \%$ ) before training to $54 \%$ (95\% CI $52 \%$ to $58 \%$ ) immediately after training (table 2 ). Scores increased for all aspects of knowledge assessed. The selfassessed Likert scale score for skills decreased from 9/18 (95\% CI 8 to 10$)$ to $7 / 18$ (95\% CI 6 to 7$)$.

Forty $(75 \%)$ PHWs were followed up at 6 months. There was no evidence of a difference between the PHWs who were and who were not reassessed in terms of baseline characteristics or total knowledge score $(p=0.46$ and $\mathrm{p}=0.33$, respectively).

Using a multilevel mixed-effects linear regression model, the baseline (before training) score was $29.9(95 \%$ CI 27.5 to 32.4). The total knowledge scores (excluding skills) increased by 11.2 points (95\% CI 8.3 to 14) immediately after training. Subsequently at 6 months post the training the increase in score was 12.4 points (95\% CI 9.2 to 15.6 , figure 3). Overall post-training scores increased but there was no evident difference in scores from immediately after training to 6 months later. There was evidence of variation between PHWs; the random effect estimate at individual level was 25.5 (95\% CI 12.5 to 52.3), and residual variance was 57.5 (95\% CI 43.3 to 76.3 ).

Table 3 gives the breakdown of the total score by the individual components of the testing. 'Case study' scores improved from a mean of $11(95 \%$ CI 9 to 13$)$ to 12 (95\% CI 7 to 15$)$ after training then to 16 (95\% CI 14 to 18) after 6 months $(\mathrm{p}<0.05)$. Likert scales assessment of 'Skills' reduced from 9 (95\% CI 8 to 10$)$ before training to $6(95 \%$ CI 6 to 7$)$ at 6 months, with 27 having lower scores after training, five having the same scores and seven having higher scores.

There was little evidence of a difference in scores $(\mathrm{p}=0.92)$ between nurses and the clinical and medical officers at baseline: nurses mean 39\% (95\% CI 35\% to $42 \%$ ) and medical officers mean $45 \%$ (95\% CI $37 \%$ to $52 \%)$ or at 6 months after training: nurses mean $56 \%$ $(95 \%$ CI $49.7 \%$ to $61.9 \%)$ and medical officers mean $57 \%(95 \%$ CI $51.9 \%$ to $61.7 \%)$.

Key findings from the interviews included that the majority of staff found the module easy to understand, with a similar level of complexity to the other IMNCI modules. Only $5(12 \%)$ reported it to be more complex and challenging. All reported they had been able to apply the skills they had learnt but some reported difficulties in

\begin{tabular}{|c|c|c|}
\hline Characteristics & $\mathbf{N}$ & $\%$ \\
\hline \multicolumn{3}{|l|}{ Demographic } \\
\hline \multicolumn{3}{|l|}{ Sex } \\
\hline Male & 18 & 33 \\
\hline Female & 36 & 67 \\
\hline \multicolumn{3}{|l|}{ Age } \\
\hline 20-30 years & 28 & 52 \\
\hline $31-40$ years & 14 & 26 \\
\hline $41-50$ years & 6 & 11 \\
\hline $51-60$ years & 4 & 7 \\
\hline Unknown & 2 & 4 \\
\hline \multicolumn{3}{|l|}{ Work } \\
\hline \multicolumn{3}{|l|}{ Clinical grade } \\
\hline Assistant medical officer & 5 & 9 \\
\hline Clinical officer & 10 & 19 \\
\hline Registered nurse & 7 & 13 \\
\hline Enrolled nurse & 30 & 56 \\
\hline Medical attendants & 2 & 4 \\
\hline \multicolumn{3}{|l|}{ Years worked } \\
\hline Less than a year & 2 & 4 \\
\hline $1-5$ years & 32 & 59 \\
\hline $5-10$ years & 8 & 15 \\
\hline More than 10 years & 10 & 19 \\
\hline Unknown & 2 & 4 \\
\hline \multicolumn{3}{|l|}{ Eye-related experience } \\
\hline \multicolumn{3}{|l|}{ Previous training in eye care } \\
\hline Yes & 3 & 6 \\
\hline No & 50 & 93 \\
\hline Unknown & 1 & 2 \\
\hline \multicolumn{3}{|l|}{ Frequency eye problems seen } \\
\hline Once a week or more & 40 & 74 \\
\hline Approximately once a month & 8 & 15 \\
\hline Less than once a month & 5 & 9 \\
\hline Unknown & 1 & 2 \\
\hline \multicolumn{3}{|l|}{ Most common eye problems } \\
\hline $\begin{array}{l}\text { Conjunctivitis/red eye in older } \\
\text { children }\end{array}$ & 35 & 65 \\
\hline Conjunctivitis in infants & 21 & 39 \\
\hline Injuries or foreign bodies & 10 & 19 \\
\hline Corneal ulcer & 2 & 4 \\
\hline Other & 2 & 4 \\
\hline \multicolumn{3}{|l|}{ Referral } \\
\hline Once a week or more & 5 & 9 \\
\hline About once a month & 15 & 28 \\
\hline Less than once a month & 32 & 59 \\
\hline Unknown & 2 & 4 \\
\hline
\end{tabular}

PHWs, primary healthcare workers. 
Table 2 Comparison of individual skills and knowledge scores before intervention and immediately after training $(\mathrm{n}=54)$

\begin{tabular}{|c|c|c|c|c|}
\hline \multirow[b]{2}{*}{ Characteristics } & \multicolumn{2}{|c|}{ Before training } & \multicolumn{2}{|c|}{ After training } \\
\hline & Mean & $95 \% \mathrm{Cl}$ & Mean & $95 \% \mathrm{Cl}$ \\
\hline MCQ score (out of 10 ) & 5 & 4 to 6 & 8 & 8 to 9 \\
\hline Best of $4 \mathrm{MCQ}$ score (out of 10 ) & 4 & 3 to 5 & 7 & 6 to 7 \\
\hline Image score (out of 19) & 10 & 9 to 11 & 14 & 13 to 15 \\
\hline Case study score (out of 30 ) & 11 & 9 to 13 & 12 & 11 to 13 \\
\hline Skills (out of 18) & 9 & 8 to 10 & 7 & 6 to 7 \\
\hline Total score excluding skills (\%) & 40 & 37 to 43 & 55 & 52 to 56 \\
\hline
\end{tabular}

$M C Q$, multiple choice question.

examining the eyes of children. Challenges they encountered were lack of community awareness about eye conditions and poor referral systems. Participants recommended more practical skills training, and video clips to assist in recognising conditions and performing procedures they were not familiar with. All except one replied that they used Arclight for red reflex testing on a regular basis. The most common changes in clinical practices were that they were examining children's eyes for the first time, referring more patients and several had reinstated ocular prophylaxis of the newborn.

\section{DISCUSSION}

Our study demonstrates the benefits of including eye health in the curriculum of healthcare workers delivering IMNCI. The advantage of including eye care in IMNCI is that it is a well-established global programme delivered by health workers who are ideally placed to implement primary eye care for young children. Including eye care in the curriculum also means it is seen as an integral component of their routine responsibilities. The IMNCI curriculum is modular, structured and symptom led which made including eye conditions relatively straightforward. The eye module follows the same structure as

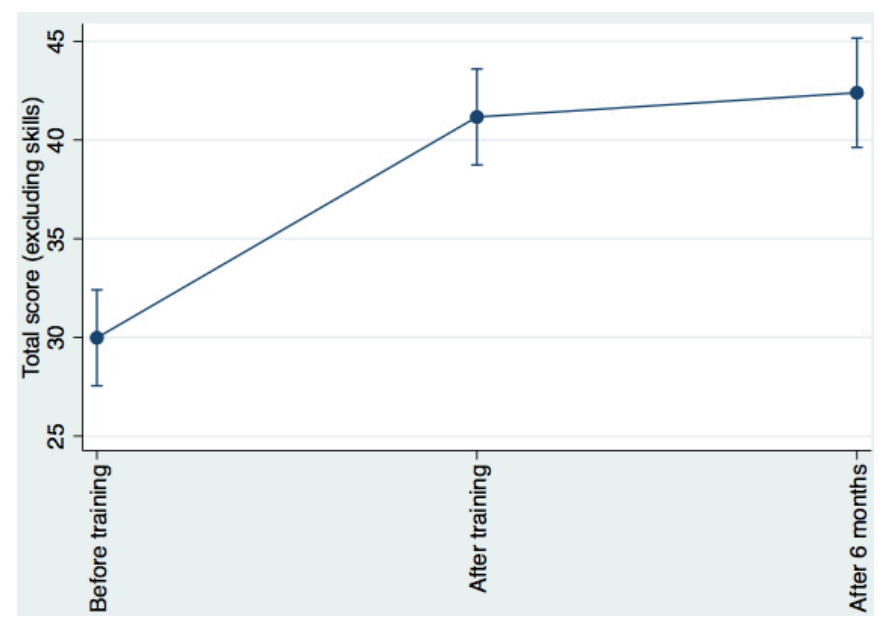

Figure 3 Change in total scores for PHWs from before training to immediately after training and 6 months after training. PHWs, primary healthcare workers other modules, indicating which specific symptoms and signs to look for, which if present lead to a management decision and how PHWs should counsel the mother. This means that the PHWs are not expected to 'diagnose' eye conditions, which overcomes the perceptions that eye conditions require higher levels of expertise than PHWs can achieve. The challenges included introducing PHWs to new skills during the short training period, overburdening them in their work, difficulty in eliciting symptoms and signs of eye disease in children and having appropriate equipment.

The module was acceptable to the PHWs who were able to include eye care into their daily practice. Most found it straightforward and at a similar level to the rest of their training. The key aspects of eye care were distilled into a familiar-looking algorithm. This ensured PHWs were not overburdened with information. The critical part of the examination is testing of the red reflex using an Arclight, which with experience, takes less than a minute to complete. The PHWs were also able to include eye care into their health talks with mothers. An educational poster for mothers/caregivers attending clinics was produced and used in this pilot study. However, the poster has not been used in subsequent training by the Ministry of Health as posters are not routine for the other modules.

The eye module and training improved knowledge scores, and the improvement was sustained over 6 months. The next step would be to demonstrate that these changes are sustained over a longer period of time, and to evaluate whether training leads to sustained change in practices and has a positive impact on children's eye health. Moreover, training alone is likely not sufficient for lasting impact at scale: reinforcement of skills, for example through routine supervision or quality improvement, is also important. ${ }^{14}$

The PHWs self-assessed confidence in diagnosing and managing eye conditions decreased after training, which might reflect a more realistic assessment and better understanding of eye problems. It has been noted previously that PHWs often have misconceptions about eye conditions, thinking they are not serious or not treatable. ${ }^{11}$ Many $\mathrm{PHW}$ requested that the training be augmented by video clips, as in other IMNCI modules. 
Table 3 Comparison of skills and knowledge scores before intervention and at 6 month follow-up $(n=40)$

\begin{tabular}{|c|c|c|c|c|c|c|}
\hline \multirow[b]{2}{*}{ Characteristics } & \multicolumn{2}{|c|}{ Before training } & \multicolumn{2}{|c|}{ After training } & \multicolumn{2}{|c|}{6 Months after training } \\
\hline & Mean & $95 \% \mathrm{Cl}$ & Mean & $95 \% \mathrm{Cl}$ & Mean & $95 \% \mathrm{Cl}$ \\
\hline MCQ score (out of 10 ) & 5 & 4 to 6 & 8 & 8 to 9 & 7 & 6 to 8 \\
\hline Best of $4 \mathrm{MCQ}$ score (out of 10 ) & 4 & 3 to 5 & 7 & 6 to 7 & 6 & 5 to 6 \\
\hline Image score (out of 19) & 10 & 9 to 11 & 14 & 13 to 15 & 14 & 13 to 15 \\
\hline Case study score (out of 30 ) & 11 & 9 to 13 & 12 & 10 to 13 & 16 & 14 to 18 \\
\hline Skills (out of 18) & 9 & 8 to 10 & 6 & 6 to 7 & 6 & 6 to 7 \\
\hline
\end{tabular}

$M C Q$, multiple choice question.

Following this study, six short videos were made to accompany the module which are now used in the training (https://www.lshtm.ac.uk/research/centres-projectsgroups/gcehp\#resources). While most PHWs found the information in the eye module to be at a similar level to the other IMNCI modules, they found examining children and eliciting the 'symptoms' and signs indicated in the eye algorithm more challenging. We anticipate that the videos will improve PHWs confidence and skills but this will require further evaluation. During the interviews PHWs did not indicate that they lacked time to examine the eyes of children, nor that this was too much to do on top of their current routine work. However, a key issue identified by PHWs was the poor referral system with lack of feedback, which is critical in enabling children to access appropriate care, and to improve PHWs learning and engagement. This was discussed with the Ministry of Health and one solution would be for the IMNCI supervisor's role to be expanded to include supporting referrals and feedback.

A limitation of the study is that we were not able to assess PHWs skills with patients and used photographs and case studies instead. This is because serious eye conditions in children are uncommon and children need to be treated promptly. While simulation models have been developed for eye care training this has tended to be for specialists examining the fundus rather than for red reflex testing.

We are aware of one study in Tanzania which is in press and another unpublished study in Nigeria in which nurses were trained to elicit the red reflex of children to detect eye conditions such as cataract. ${ }^{15}$ In one study three devices were assessed, one of which was the Arclight. Both studies demonstrated that nurses can correctly and reliably undertake this screening test (personal communications). However, neither study addressed the need for comprehensive primary eye care for children, nor how red reflex testing could be integrated into routine care for children. One of the key challenges has been ensuring eye care (including red reflex testing) is part of routine child healthcare programmes for children. The importance of integrating eye care into the IMNCI programme is that it becomes sustainable and scalable at national and global levels, rather than dependant on short-term funding in vertical programmes.
During the study, the eye module was adopted for scale-up by the Ministry of Health in Tanzania and is now part of routine IMNCI training. To date over 3000 PHWs delivering IMNCI have been trained in child eye health. The African regional office for WHO has recently published a primary eye care training manual for all ages, which includes an algorithm for managing eye conditions in children. ${ }^{16}$ It is important to build on this integrated training programme towards a comprehensive primary eye care approach for children under 5 years of age. Our study has shown that eye care can be integrated into a routine child health programme at primary care level which could address the gap in primary child eye care in LMICs. A larger scale evaluation of training is required, alongside enhancing supervisory support, to assess if PHWs are able to include the knowledge and skills from the eye module into their routine practice and ultimately improve child health.

\section{Twitter Aeesha N J Malik @aeeshamalik}

Acknowledgements Dr Naibu Mkongwa, Ministry of Health, Dr Frank Albert, Dr Jacinta Feksi and all the members of the steering committee in Tanzania.

Contributors ANJM, MM and CG conceptualised and designed the study. ANM and MM led the collection of data. All authors analysed the data. MJK undertook the statistical analysis of the data. ANM wrote the first draft and revised the manuscript with all authors contributing to revisions.

Funding This work was supported by the British Council for Prevention of Blindness (BCPB) grant number 14836 and CBM grant number 3324-MYP.

Competing interests None declared.

Patient consent for publication Not required.

Ethics approval Ethical approval was obtained from LSHTM Ethics Committee (Ref: 13484), MUHAS Ethics committee (Ref: 2017-12-21/AEC/Vol.XI//84) and the Tanzania National Institute for Medical Research (Ref: NIMR/HQ/R.8a/Nol.IX/2622). Permission to conduct the study was given by the Regional and District Executive Secretaries. All PHWs gave written informed consent to participate.

Provenance and peer review Not commissioned; externally peer reviewed.

Data availability statement Data are available upon reasonable request. All data relevant to the study are included in the article or uploaded as supplementary information. Further deidentified participant data are available by email to: aeesha. malik@Ishtm.ac.uk.

Open access This is an open access article distributed in accordance with the Creative Commons Attribution Non Commercial (CC BY-NC 4.0) license, which permits others to distribute, remix, adapt, build upon this work noncommercially, and license their derivative works on different terms, provided the original work is properly cited, appropriate credit is given, any changes made indicated, and the use is non-commercial. See: http://creativecommons.org/ licenses/by-nc/4.0/. 
ORCID iD

Aeesha N J Malik http://orcid.org/0000-0002-9379-4350

\section{REFERENCES}

1 Rahi JG CE. Epidemiology of visual impairment in children. 4th edn. Elsevier, 2016.

2 World Health Organisation. Universal eye health: a global action plan 2014-2019, 2013. Available: http://whoint/blindness/actionplan/en/

3 Gilbert C, Foster A. Childhood blindness in the context of VISION 2020-the right to sight. Bull World Health Organ 2001;79:227-32.

4 World Health Organization. A five year project for the prevention of childhood blindness: report of a who consultation. World Health Organization, 2002.

5 World Health Organization. Vision 2020 global initiative for the elimination of avoidable blindness action plan 2006-2011. 2007.

6 World Health Organization. Ten years in public health, 2007-2017: report by DR Margaret Chan, Director-General 2017.

7 World Health Organisation,. International Agency for the Prevention of Blindness. Preventing blindness in children : report of a WHO/ IAPB scientific meeting, Hyderabad, India, 13-17 April 19992020.

8 Malik ANJ, Mafwiri M, Gilbert C. Integrating primary eye care into global child health policies. Arch Dis Child 2018;103:176-80.

9 World Health Organization. Integrated management of childhood illness, 2017. Available: http://wwwwhoint/maternal child adolescent/topics/child/imci/en/
10 UNICEF. UNICEF Republic of Tanzania data. Available: https:// datauniceforg/country/tza/

11 Mafwiri MM, Kisenge R, Gilbert CE. A pilot study to evaluate incorporating eye care for children into reproductive and child health services in Dar-es-Salaam, Tanzania: a historical comparison study. BMC Nurs 2014;13:15.

12 Blaikie A, Sandford-Smith J, Tuteja SY, et al. Arclight: a pocket ophthalmoscope for the 21st century. BMJ 2016;355:i6637.

13 World Health Organization. IMCl set of distance learning modules, 2014. Available: https://wwwwhoint/maternal_child_adolescent/ documents/9789241506823/en/

14 Rowe AK, Rowe SY, Peters DH, et al. Effectiveness of strategies to improve health-care provider practices in low-income and middle-income countries: a systematic review. Lancet Glob Health 2018:6:e1163-75.

15 Mndeme FG, Mmbaga BT, Kim MJ, et al. Red reflex examination in reproductive and child health clinics for early detection of paediatric cataract and ocular media disorders: cross-sectional diagnostic accuracy and feasibility studies from Kilimanjaro, Tanzania. Eye 2020. doi:10.1038/s41433-020-1019-5. [Epub ahead of print: 16 Jun 2020].

16 World Health Organization. Brazzaville, Regional Office for Africa. PRIMARY EYE CARE TRAINING MANUAL - A course to strengthen the capacity of health personnel to manage eye patients at primarylevel health facilities in the African Region, 2018. Available: https:// www.afro.who.int/sites/default/files/2018-06/WEB-2835-OMS-AfroPrimaryEyeCaretrainingmanual-20180406.pdf 